\title{
In vivo growth of transitional and renal cell carcinoma cell lines can be suppressed by the adenovirus-mediated expression of a soluble form of vascular endothelial growth factor receptor
}

\author{
HARUHIKO ICHIKURA ${ }^{1 *}$, MASATOSHI ETO ${ }^{1 *}$, HIKARU UENO ${ }^{3}$, MAMORU HARADA $^{4}$, \\ KOICHI TAKAYAMA ${ }^{2}$, NORIAKI TOKUDA ${ }^{1}$, KATSUNORI TATSUGAMI ${ }^{1}$ and SEIJI NAITO $^{1}$
}

\begin{abstract}
Department of ${ }^{1}$ Urology and ${ }^{2}$ Research Institute for Diseases of the Chest, Graduate School of Medical Sciences, Kyushu University, 3-1-1 Maidashi, Higashi-ku, Fukuoka 812-8582; ${ }^{3}$ Department of Biochemistry and Molecular Pathophysiology, University of Occupational and Environmental Health, School of Medicine, 1-1 Iseigaoka, Yahatanishi-ku, Kitakyushu 807-8555; ${ }^{4}$ Department of Immunology, Kurume University School of Medicine, 67 Asahi-machi, Kurume 830-0011, Japan
\end{abstract}

Received October 19, 2005; Accepted January 29, 2006

\begin{abstract}
Antiangiogenic therapy is a promising strategy for the treatment of cancer since tumor development and metastases require angiogenesis. Vascular endothelial growth factor (VEGF) is one of the most important factors in tumor angiogenesis. In the present study, we investigated the antitumor effect of an adenovirus (AdVEGF-ExR) expressing the extracellular domain of the human VEGF receptor (flt- 1 ) using two different urological tumor/mouse systems. RENCA, a renal cell carcinoma of BALB/c origin, and MBT-2, a poorly differentiated transitional carcinoma of $\mathrm{C} 3 \mathrm{H} / \mathrm{He}$ origin, were used. Both types of tumor were in vitro infected with AdVEGFExR and inoculated subcutaneously into the abdomens of syngenenic mice, and tumor growth was measured twice weekly. In some experiments, BALB/c mice with established RENCA tumors were injected intramuscularly with AdVEGFExR as a therapeutic model. The cytotoxicity of spleen cells from the tumor-rejected mice was assessed by ${ }^{51} \mathrm{Cr}$-release assay. Although the in vitro cell growth of either MBT-2 or RENCA was not affected by infection with AdVEGF-ExR, the in vivo growth of both AdVEGF-ExR-infected tumors was significantly suppressed in the syngeneic mice. In addition,
\end{abstract}

Correspondence to: Dr Masatoshi Eto, Department of Urology, Graduate School of Medical Sciences, Kyushu University, 3-1-1 Maidashi, Higashi-ku, Fukuoka 812-8582, Japan

E-mail: etom@uro.med.kyushu-u.ac.jp

${ }^{*}$ Contributed equally

Abbreviations: CTLs, cytotoxic T lymphocytes; IL, interleukin; MOI, multiplicity of infection; PFU, plaque-forming unit; VEGF, vascular endothelial growth factor

Key words: gene therapy, vascular endothelial growth factor receptor, flt-1, carcinoma, renal cell, bladder tumor although 2 of 5 mice rejected the AdVEGF-ExR-infected RENCA, tumor-specific cytotoxic T lymphocytes were not generated from their spleen cells, thus suggesting no cellular immune response. In a therapeutic model, intramuscular injections of AdVEGF-ExR at a remote site also significantly suppressed the growth of the subcutaneously established RENCA. These results indicate that the adenovirus-mediated expression of a soluble VEGF receptor can be an effective therapy for urological cancer treatment; however, such VEGFtargeted gene therapy is not necessarily accompanied by subsequent antitumor $\mathrm{T}$ cell immunity.

\section{Introduction}

Angiogenesis is required for various physiological and pathological events, including tumor development and metastases $(1,2)$. For tumor angiogenesis, vascular endothelial growth factor (VEGF) may be one of the most important factors. Cancer cells have been suggested to produce VEGF and induce angiogenesis through a paracrine loop (3). Therefore, antiangiogeneic therapy targeting VEGF is a promising strategy for cancer treatment (4). Tumor suppression has been confirmed in animal experiments by targeting VEGF or its receptor (4-8). We have also reported that the adenovirusmediated expression of a soluble form of VEGF receptor (flt- 1 ) can effectively suppress in vivo tumor growth of human cancer cells in nude mice (9). In most cases, however, the antiangiogenic effect has been investigated by experiments in which xenogeneic human cancer cells were transplanted into SCID or nude mice (5-10).

In this study, we determined whether or not the adenovirusmediated expression of a soluble form of VEGF receptor could effectively suppress in vivo tumor growth in syngeneic mice, using two different murine urological tumors. Our results indicate that the overexpression of VEGF receptor in tumor cells or at a remote site resulted in a significant suppression of in vivo tumor growth in syngeneic mice, but VEGFtargeted gene therapy is not necessarily accompanied by subsequent antitumor $\mathrm{T}$ cell immunity. 


\section{Materials and methods}

Preparation of adenoviral vectors. Replication-defective E1 and E3- adenoviral vectors expressing either the entire ectodomain of the human VEGF receptor (flt-1) fused to the Fc portion of human IgG (AdVEGF-ExR) or bacterial B-galactosidase (AdLacZ), as a control, were prepared as described previously $(11,12)$. A CA promoter comprising a cytomegalovirus enhancer and a chicken $ß$-actin promoter was used for the expression of the genes. The titer of the virus stock was assessed by a plaque formation assay using 293 cells, and the titer was expressed in plaque-forming units (PFUs).

Mice and tumor cell lines. Female BALB/c and $\mathrm{C} 3 \mathrm{H} / \mathrm{He}$ mice were obtained from Japan SLC (Shizuoka, Japan). All mice were kept in specific pathogen-free conditions and used for experiments at 8 weeks of age. RENCA and colon 26 are renal and colon carcinoma cell lines of $\mathrm{BALB} / \mathrm{c}$ origin, respectively. MBT-2 is a poorly differentiated transitional carcinoma cell line of $\mathrm{C} 3 \mathrm{H} / \mathrm{He}$ origin. All tumor lines were maintained in vitro in complete culture medium consisting of RPMI-1640 (Gibco, Grand Island, NY) supplemented with $10 \%$ heat-inactivated fetal calf serum (HyClone, Logan, UT), $50 \mu \mathrm{M}$ 2-mercaptoethanol, $20 \mathrm{mM}$ HEPES, $30 \mu \mathrm{g} / \mathrm{ml}$ gentamicin (Schering Corporation, Kenilworth, NJ), and $0.2 \%$ sodium bicarbonate.

Measurement of VEGF. Confluent tumor cells were cultured for $24 \mathrm{~h}$ and then the culture medium was collected. After centrifugation, the supernatant was stored at $-80^{\circ} \mathrm{C}$ until assay. The VEGF protein in the culture medium was detected using an ELISA kit (Immuno Biological Laboratories, Tokyo, Japan) according to the manufacturer's instructions.

Assay of in vivo tumor growth. In vitro gene transfer into tumor cells was carried out by incubation with the adenoviral vector in a serum-free medium (RPMI-1640 containing $0.05 \%$ BSA, $1 \mu \mathrm{g} / \mathrm{ml}$ insulin, $5 \mu \mathrm{g} / \mathrm{ml}$ transferrin, and $25 \mathrm{mmol} / \mathrm{liter}$ HEPES $\mathrm{pH}$ 7.4) for $2 \mathrm{~h}$ at room temperature with gentle agitation, as described previously (13). To confirm the gene transfer efficiency by an adenoviral vector, RENCA and MBT2 cells infected with various MOI of AdLacZ (MOI 0-200) were stained with 5-bromo-4-chloro-3-indolyl- $\beta$ galactopyranoside (X-gal) and the number of blue-stained cells was counted. Based on the results of the gene transfection efficiency assay, MBT-2 cells were infected with AdVEGFExR (MOI 100) or AdLacZ (MOI 100), and 2.5x106 MBT-2 cells were inoculated subcutaneously into the abdomens of syngeneic $\mathrm{C} 3 \mathrm{H} / \mathrm{He}$ mice. In addition, RENCA cells were infected with AdVEGF-ExR (MOI 200) or AdLacZ (MOI 200 ), and $5 \times 10^{5}$ RENCA cells were inoculated subcutaneously into syngeneic BALB/c mice. Thereafter, two diameters of subcutaneous tumors at right angles were measured twice weekly with calipers, and the product of the two values was shown as the tumor size $\left(\mathrm{mm}^{2}\right)$.

Assay of cytotoxicity. An assay of cytotoxicity was carried out, as reported previously (14). In brief, spleen cells from tumor-rejected mice were in vitro stimulated with MMCinactivated tumor cells for 4 days in the presence of $20 \mathrm{U} / \mathrm{ml}$

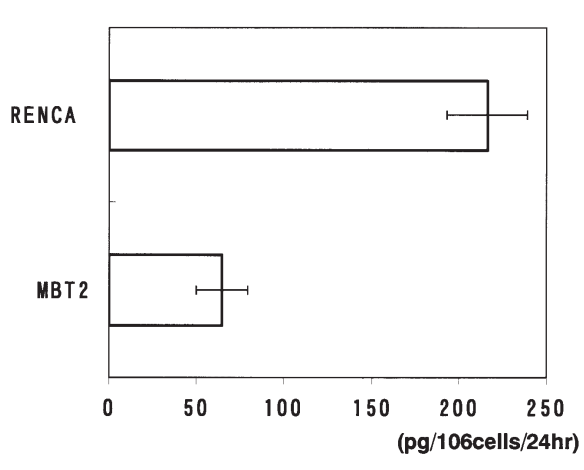

Figure 1. VEGF production by RENCA and MBT-2 cell lines. The amounts of VEGF in the culture medium, which were prepared from RENCA and MBT-2 cell lines ( $1 \times 10^{6}$ cells/24 h), were measured using ELISA as described in Materials and methods.

of IL-2. Thereafter, these cultured cells were harvested, and tested for cytotoxicity against both the RENCA and colon 26 cell lines by a $4-\mathrm{h}{ }^{51} \mathrm{Cr}$-release assay. The ${ }^{51} \mathrm{Cr}$-labelled target cells $\left(1 \times 10^{4}\right.$ cells/well $)$ were cultured with effector cells in round 96-well plates at the indicated effector/target ratios. The specific ${ }^{51} \mathrm{Cr}$-release was calculated according to the formula: (test cpm - spontaneous cpm) x100/(total cpm spontaneous cpm). Spontaneous release was determined by the supernatant of the sample incubated with no effector cells, and the total release was then determined by the supernatant of the sample incubated with $1 \%$ Triton X (Wako Pure Chemical Industries, Osaka, Japan).

Therapeutic model. BALB/c mice were subcutaneously inoculated with RENCA cells $\left(5 \times 10^{5}\right.$ cells $)$. After the establishment of the tumors, these mice were also injected with AdVEGF-ExR ( $5 \times 10^{8}$ PFU) twice on days 10 and 17, in the femoral muscle. As a control, LdLacZ $\left(5 \times 10^{8} \mathrm{PFU}\right)$ was administered. The amount of soluble VEGF receptor, which was tagged with the Fc portion of human $\mathrm{IgG}$, in the serum of the mice was measured by ELISA using an antihuman $\mathrm{IgG}$ antibody, as described previously (9). Two diameters of subcutaneous tumors at right angles were measured twice weekly with calipers, and the product of the two values was shown as the tumor size $\left(\mathrm{mm}^{2}\right)$.

Statistics. The statistical significance of the data was determined using the unpaired two-tailed Student's t-test. A $\mathrm{P}$-value $<0.05$ was considered to be statistically significant.

\section{Results}

VEGF production by renal and transitional cell carcinoma cell lines. Before investigating the antitumor effect of the soluble VEGF receptor on two different murine urological tumor cell lines, a RENCA renal cell carcinoma and an MBT-2 bladder carcinoma, we determined whether or not these tumor cells themselves could secrete VEGF. Both tumor cell lines were found to produce substantial levels of VEGF (Fig. 1). No VEGF was detected in the culture medium without tumor cells (data not shown). These results suggest that both murine tumor cell lines might utilize VEGF in their tumor angiogenesis in vivo. 

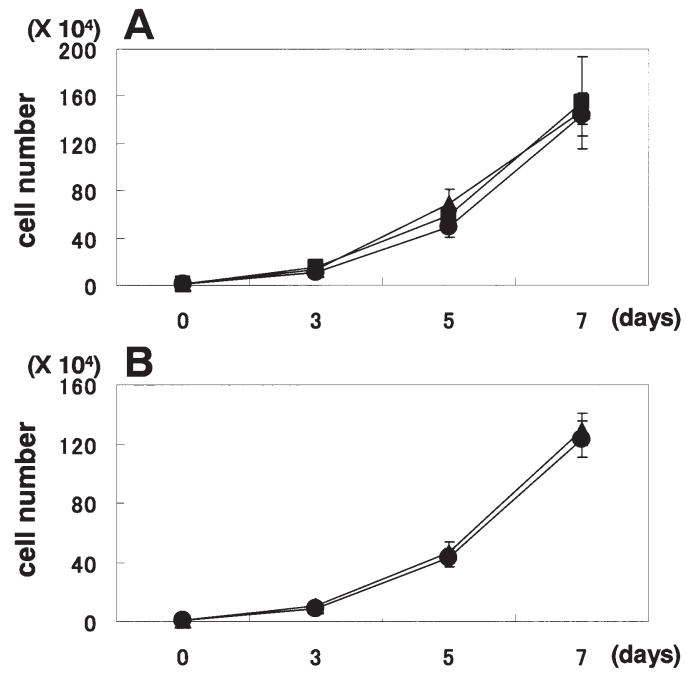

Figure 2. No effect of the infection with AdVEGF-ExR on in vitro growth of either MBT-2 or RENCA cells. (A) MBT-2 cells were infected with either (•) AdVEGF-ExR (MOI 20), (•) AdLacZ (MOI 20), or (^) no adenoviral vector, and then their in vitro growth was examined. $1 \times 10^{4} \mathrm{MBT}$ tumor cells were applied to each well and the cell number was counted on the indicated days. The mean of three determinants is shown. (B) Similarly,

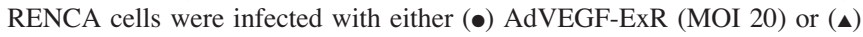
no adenoviral vector, and then their in vitro growth was examined.

Infection with AdVEGF-ExR had no effect on in vitro tumor growth. To transfer the gene encoding human VEGF receptor, we constructed an adenovirus (AdVEGF-ExR) expressing the entire cellular domain of the human VEGF receptor fused to the Fc portion of human $\operatorname{IgG}$, as previously reported $(11,12)$. We then determined whether an infection with AdVEGFExR could have any influence on the in vitro growth of tumor cell lines. As shown in Fig. 2A, no difference was observed in the in vitro growth of MBT-2 cells after infection with either AdVEGF-ExR or AdLacZ. In addition, no difference was observed in the growth of RENCA cells after infection with or without AdVEGF-ExR (Fig. 2B).

In vivo growth suppression of carcinoma cells after the gene transfer of VEGF receptor. We next determined whether infection with AdVEGF-ExR has any effect on in vivo tumor growth using two different tumor/mouse systems. As shown in Fig. 3A, the in vivo tumor growth of MBT-2 in syngeneic $\mathrm{C} 3 \mathrm{H} / \mathrm{He}$ mice was significantly suppressed when these tumor cells were pre-infected with AdVEGF-ExR compared to that of AdLacZ-infected MBT-2. However, no mice rejected the challenged AdVEGF-ExR-infected MBT-2 cells. On the other hand, the in vivo tumor growth of AdVEGF-ExR-infected RENCA was also significantly suppressed compared to that of AdLacZ-infected RENCA cells (Fig. 3B). Two of five BALB/c mice rejected the challenged AdVEGF-ExR-infected RENCA cells.

Although there have been many reports investigating the antitumor effect of VEGF-targeting therapy (4-8), whether or not VEGF-targeted gene therapy could elicit a subsequent cellular immunity has yet to be determined. In addition, as we used immunocompetent mice and syngeneic tumors, but not nude mice with xenogeneic tumors, we wanted to examine acquired immunity after the VEGF-targeted gene therapy. RENCA-specific cytotoxic T lymphocytes have been reported
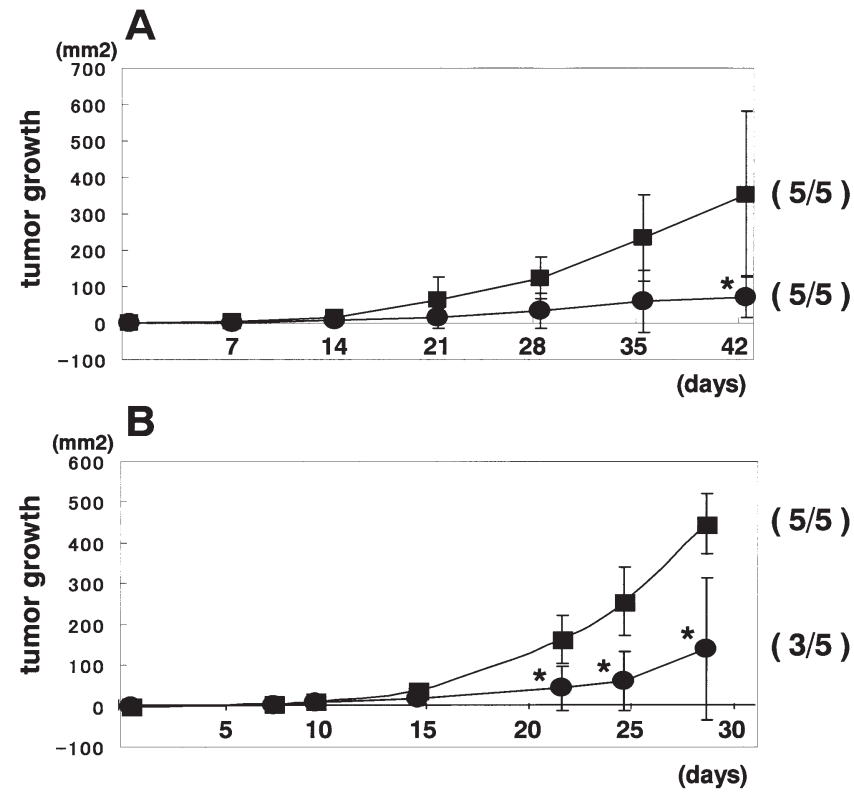

$(5 / 5)$

Figure 3. Suppression of in vivo tumor growth by infection with AdVEGFExR. (A) Based on the results of the gene transfection efficiency assay described in Materials and methods, MBT-2 cells were infected with $(\bullet)$ AdVEGF-ExR (MOI 100) or (๘) AdLacZ (MOI 100), and 2.5x106 MBT-2 cells were inoculated subcutaneously into syngeneic $\mathrm{C} 3 \mathrm{H} / \mathrm{He}$ mice. (B) RENCA cells were infected with (•) AdVEGF-ExR (MOI 200) or (घ) AdLacZ (MOI 200 ), and $5 \times 10^{5}$ RENCA cells were inoculated subcutaneously into syngeneic $\mathrm{BALB} / \mathrm{c}$ mice. In both experiments, each group consisted of 5 mice, and their mean findings are shown. The number in parentheses shows the mice with tumors/all mice on the final observation day in each experiment. Similar results were obtained in two other experiments. ${ }^{*} \mathrm{P}<0.05$ statistically significant.

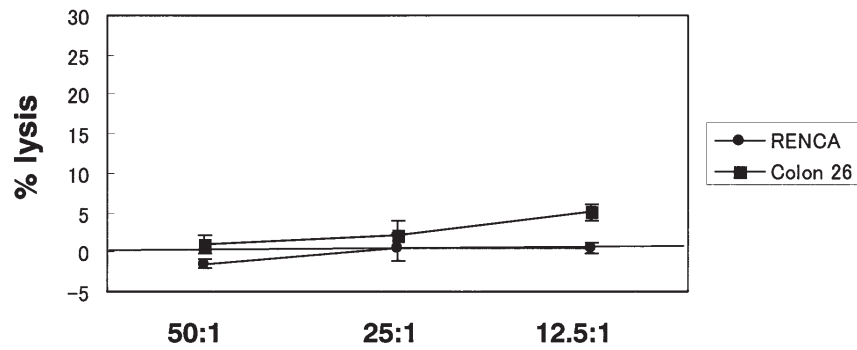

Figure 4. No generation of RENCA-specific CTLs was observed in the mice that rejected the AdVEGF-ExR-infected RENCA cells. Spleen cells were taken 5 weeks after the tumor inoculation from the BALB/c mouse, which rejected the AdVEGF-ExR-infected RENCA, and in vitro stimulated with inactivated RENCA cells in the presence of IL-2 $(20 \mathrm{U} / \mathrm{ml})$. Their cytotoxicity against both RENCA and colon 26 was then examined by a $4-\mathrm{h}{ }^{51} \mathrm{Cr}$-release assay. Similar results were obtained in two other experiments using BALB/c mice that rejected the AdVEGF-ExR-infected RENCA.

to be generated from immunized mice (15). We also detected RENCA-specific interferon- $\gamma$ production in the spleen cells of $\mathrm{BALB} / \mathrm{c}$ mice that rejected the RENCA tumors in other experimental models (16). We therefore attempted to generate RENCA-specific cytotoxic T lymphocytes (CTLs) from the spleen cells of BALB/c mice that rejected the AdVEGF-ExRinfected RENCA cells. Spleen cells were taken 5 weeks after tumor inoculation. We did not assess RENCA-specific CTLs in the control BALB/c mice that were subcutaneously injected with AdLacZ-infected RENCA cells, as nearly all of the mice in that group were already dead 5 weeks after tumor inoculation 


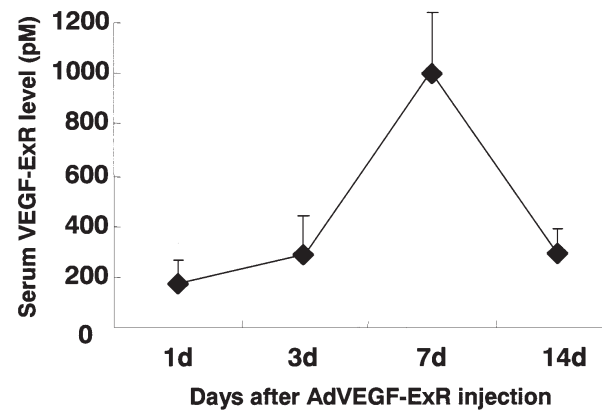

Figure 5. The soluble VEGF receptor was detectable in the circulating blood after an intramuscular injection of AdVEGF-ExR. After intramuscular injection of AdVEGF-ExR $\left(5 \times 10^{8} \mathrm{pfu}\right)$, the soluble VEGF receptor in the circulating blood was quantified by ELISA. The mean \pm SD from 4 individual mice is shown.

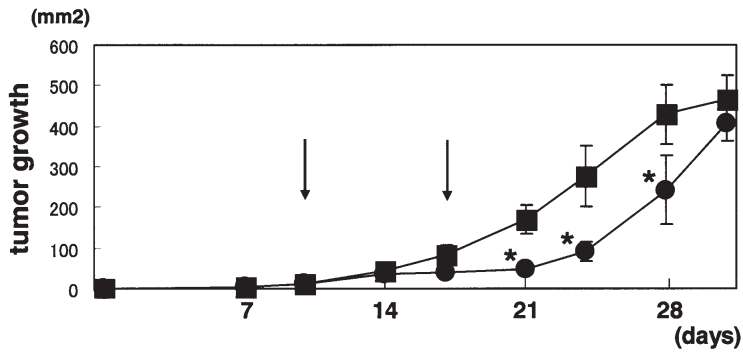

Figure 6. In vivo growth suppression of RENCA cells by VEGF-ExR production at a remote site. RENCA $\left(5 \times 10^{5}\right.$ cells $)$ was injected subcutaneously into the abdomens of syngeneic BALB/c mice on day 0 . After the establishment of tumors, these mice received two injections of either $(\bullet)$ AdVEGF-

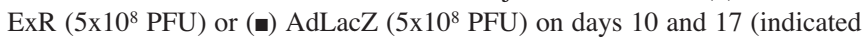
with black arrows) in the femoral muscle. Tumor size was inspected twice weekly. Each group consisted of 5 mice, and their mean is shown. Similar results were obtained in two other experiments. ${ }^{*} \mathrm{P}<0.05$ statistically significant.

due to tumor progression (data not shown). However, no RENCA-specific CTLs were induced, even in the BALB/c mice that rejected the AdVEGF-ExR-infected RENCA cells (Fig. 4).

In vivo growth suppression of RENCA by VEGF-ExR production at a remote site. It is therapeutically important to determine whether the suppression of tumor growth can be induced even when AdVEGF-ExR is administered to a remote site because it is impossible to deliver AdVEGF-ExR to all cancer cells in vivo. We initially quantified the soluble VEGF receptor in the circulating blood using ELISA after an intramuscular injection of AdVEGF-ExR ( $\left.5 \times 10^{8} \mathrm{PFU}\right)$. Similar to our previous report using nude mice with xenografts (9), the intramuscular administration of AdVEGF-ExR (5x10 8 PFU) generated considerable amounts of the soluble VEGF receptor in the blood (Fig. 5). The serum level of the soluble receptor peaked on day 7 after administration and declined thereafter (Fig. 5). However, it was still detectable on day 14 (Fig. 5). The BALB/c mice were then inoculated subcutaneously with RENCA cells, and they were intramuscularly injected with AdVEGF-ExR $\left(5 \times 10^{8}\right.$ PFU) twice after the establishment of tumors. As a result, the intramuscular injections of AdVEGFExR significantly suppressed the growth of subcutaneously established RENCA in comparison to the mice injected with
AdLacZ (Fig. 6). This result indicates that the growth of RENCA cells in syngeneic BALB/c mice can be suppressed by VEGF-targeted gene therapy, even when the soluble form of VEGF receptor is produced at a remote site.

\section{Discussion}

We previously reported that the in vivo growth of human tumor cell lines in nude mice can be suppressed by the adenovirus-mediated overexpression of the soluble form of VEGF receptor (9). Other researchers have also reported the antitumor effect of soluble VEGF receptor $(4,7,8)$. In this study, we investigated whether the antitumor effect by the gene transfer of soluble VEGF receptor could suppress the in vivo growth of two different urological tumor cell lines in syngeneic mice. As a result, the in vivo growth of transitional and renal cell carcinoma cell lines was significantly suppressed by an overexpression of the soluble form of VEGF receptor in tumor cells. Forty percent of the mice rejected the VEGF receptor-expressing RENCA. In addition, in a therapeutic model, the in vivo growth of subcutaneously established RENCA cells was significantly suppressed by the gene transfer of the VEGF receptor at a remote site. This means that a circulating VEGF receptor could have an antitumor effect on established tumors. Due to the present inability to effectively deliver targeting genes into all cancer cells in vivo, this result is therapeutically important. These lines of evidence indicate that the adenovirus-mediated expression of the soluble VEGF receptor can be an effective therapy for urological cancer treatment.

Another crucial finding in this study is that no apparent antitumor cellular immunity was elicited in mice that rejected the VEGF receptor-expressing RENCA. This finding indicates that the VEGF-targeting gene therapy is not necessarily associated with the subsequent priming of antitumor CTLs, and suggests that the observed antitumor effect simply reflects the antiangiogenic effect induced by the soluble VEGF receptor. In this sense, our results are compatible with those of Boehm et al who showed that repeated treatments with endostatin, a potent angiogenesis inhibitor, induced a prolonged tumor dormancy without acquired immunity to original tumors (17). Furthermore, the antitumor effect observed when AdVEGF-ExR-infected RENCA cells were injected seems to reflect the local expression of the soluble VEGF receptor, as no soluble VEGF receptor was ever detected in the circulating blood after tumor inoculation (data not shown). Although we have not investigated whether overexpression of the soluble form of VEGF receptor in urological cancer cell lines resulted in apoptosis or necrosis in vivo, apoptosis was observed in the AdVEGF-ExR-infected human cancer cells in SCID mice (9). Previous reports suggest that necrotic cancer cells can activate stimulatory dendritic cells and prime antigen-specific CTLs more efficiently than apoptotic cancer cells $(18,19)$. Therefore, apoptotic RENCA cells might not efficiently activate stimulatory dendritic cells. Otherwise, the amount of tumor antigens derived from the challenged AdVEGF-ExRinfected RENCA cells might be insufficient to elicit acquired immunity. Further experiments are needed to elucidate the underlying mechanism of this observation.

Antiangiogenic therapy is a promising strategy for cancer treatment since tumor development requires angiogenesis 
$(1,2)$. Indeed, Yang et al reported that bevacizumab, a neutralizing antibody against vascular endothelial growth factor, significantly prolonged the time to progression of disease in patients with metastatic renal cell carcinoma (20). In our study, however, although the therapeutic model with gene transfer of the VEGF receptor at a remote site demonstrated a significant delay in the in vivo growth of subcutaneously established RENCA cells, none of the mice rejected the established tumors and the treated tumors reached a size similar to that of the control tumors at the last data point (Fig. 6). This result may show the limitations of antiangiogenic therapy used alone. If gene therapy targeting tumor angiogenesis is associated with subsequent antitumor cellular immunity, an antitumor effect could be synergistically induced in tumor-bearing hosts. Although we utilized an adenovirus to transfer the targeting gene into cancer cells or tumor-bearing hosts, the repeated administration of adenovirus is very limited because of the induction of neutralizing antibody against adenovirus-derived antigens (21). In addition, the induction of antibody against the Fc portion of human $\mathrm{IgG}$ in our vector may also limit the repeated administration of our vector. As a result, we only performed two intramuscular injections of AdVEGF-ExR when AdVEGF-ExR was administered at a remote site from the tumor cells. We suppose that antiangiogenic therapy and antitumor immunotherapy are not mutually exclusive, and the development of new therapeutic modalities that can both induce antiangiogenesis and antitumor $\mathrm{T}$ cell immunity could dramatically improve the antitumor effect in tumor-bearing hosts.

The limitation of this study is that we used a subcutaneous injection of tumor cells. The organ-specific factors involved in tumor growth are absent in this method even though we used two urological tumors. The reason we used a subcutaneous injection was so that we could easily monitor tumor growth in vivo. Many reports, including our previous study, also used a subcutaneous injection of tumor cells $(22,23)$. We thus believe that orthotopic tumor models should be used in future to assess the features of urological tumors more precisely.

In conclusion, using murine models, our results show that the adenovirus-mediated expression of a soluble VEGF receptor can be an effective therapy for urological cancer treatment. However, such VEGF-targeted gene therapy is not necessarily accompanied by any subsequent antitumor $\mathrm{T}$ cell immunity, implying the possibility of an improved antitumor effect with both antiangiogenic therapy and antitumor immunotherapy.

\section{Acknowledgements}

This work was supported in part by the Japan Society for the Promotion of Science, a Grant-in-Aid for Scientific Research (no. 16659440 to M.E.), a Grant-in-Aid for Cancer Research from the Fukuoka Cancer Society, Fukuoka, Japan (to M.E.), and a grant from the Suzuki Foundation for Urological Medicine (to M.E.).

\section{References}

1. Folkman J and Shing Y: Angiogenesis. J Biol Chem 267: 10931-10934, 1992.

2. Folkman J: Angiogenesis in cancer, vascular, rheumatoid and other disease. Nature Med 1: 27-31, 1995.
3. Ferrara N, Houck K, Jakeman L and Leung DW: Molecular and biological properties of the vascular endothelial growth factor family of proteins. Endocr Rev 13: 18-32, 1992.

4. Kuo CJ, Farnebo F, Yu EY, et al: Comparative evaluation of the antitumor activity of antiangiogenic proteins delivered by gene transfer. Proc Natl Acad Sci USA 98: 4605-4610, 2001.

5. Skobe M, Rockwell P, Goldstein N, Vosseler S and Fusenig NE: Halting angiogenesis suppress carcinoma cell invasion. Nature Med 3: 1222-1227, 1997.

6. Cheng SY, Huang HJS, Nagane M, et al: Suppression of glioblastoma angiogenicity and tumorigenicity by inhibition of endogenous expression of vascular endothelial growth factor. Proc Natl Acad Sci USA 93: 8502-8507, 1996.

7. Millauer B, Shawver LK, Plate KH, Risau W and Ullrich A: Glioblastoma growth inhibited in vivo by a dominant negative FLK-1 mutant. Nature 367: 576-579, 1994.

8. Goldman CK, Kendall RL, Cabrera G, et al: Paracrine expression of a native soluble vascular endothelial growth factor receptor inhibits tumor growth, metastasis, and mortality rate. Proc Natl Acad Sci USA 95: 8795-8800, 1998.

9. Takayama K, Ueno H, Nakanishi Y, et al: Suppression of tumor angiogenesis and growth by gene transfer of a soluble form of vascular endothelial growth factor receptor into a remote organ. Cancer Res 60: 2169-2177, 2000.

10. Davidoff AM, Nathwani AC, Spurbeck WW, Ng CY, Zhou J and Vanin EF: rAAV-mediated long-term liver-generated expression of an angiogenesis inhibitor can restrict renal tumor growth in mice. Cancer Res 62: 3077-3083, 2002.

11. Ueno H, Li JJ, Tomita H, et al: Quantitative analysis of repeated adenovirus-mediated gene transfer into injured canine femoral arteries. Arterioscler Thromb Vesc Biol 15: 2246-2253, 1995.

12. Miyake S, Makimura M, Kanegae Y, et al: Efficient generation of recombinant adenoviruses using adenovirus DNA-terminal protein complex and a cosmid bearing the full-length virus genome. Proc Natl Acad Sci USA 93: 1320-1324, 1996.

13. Yamamoto H, Ueno H, Ooshima A and Takeshita A: Adenovirusmediated transfer of a truncated transforming growth factor (TGF)- $\beta$ type II receptor completely and specifically abolished diverse signaling by TGF- $\beta$ in vascular wall cells in primary culture. J Biol Chem 271: 16253-16259, 1996.

14. Eto M, Harada M, Tamada K, et al: Antitumor activity of interleukin-12 against murine bladder cancer. J Urol 163: 1549-1552, 2000.

15. Seki N, Brooks AD, Carter CRD, et al: Tumor-specific CTLs kill murine renal cancer cells using both perforin and fas ligandmediated lysis in vitro, but cause tumor regression in vivo in the absence of perforin. J Immunol 168: 3484-3492, 2002.

16. Harano M, Eto M, Iwai T, et al: Renal cancer treatment with low levels of mixed chimerism induced by nonmyeloablative regimen using cyclophosphamide in mice. Cancer Res 65: 1003210040, 2005

17. Boehm T, Folkman J, Browder T and O'Reilly MS: Antiangiogenic therapy of experimental cancer does not induce acquired drug resistance. Nature 390: 404-407, 1997.

18. Gallucci S, Lolema M, and Matzinger P: Natural adjuvants: endogenous activators of dendritic cells. Nature Med 5: 1249-1255, 1999.

19. Sauter B, Albert ML, Francisco L, Larsson M, Somersan S and Bhardwaj N: Consequences of cell death: exposure to necrotic tumor cells, but not primary tissue cells or apoptotic cells, induces the maturation of immunostimulatory dendritic cells. J Exp Med 191: 423-434, 2000.

20. Yang JC, Haworth L, Sherry RM, et al: A randomized trial of bevacizumab, an anti-vascular endothelial growth factor antibody, for metastatic renal cancer. N Engl J Med 349: 427-434, 2003.

21. Rosenberg SA, Zhai Y, Yang JC, et al: Immunizing patients with metastatic melanoma using recombinant adenoviruses encoding MART-1 or gp100 melanoma antigens. J Natl Cancer Inst 90: 1894-1900, 1998.

22. Eto M, Harada M, Tatsugami K, et al: Anti-tumour activity of heat-shock protein 60 -recognizing $\mathrm{CD}^{+} \mathrm{T}$ cells against syngeneic murine renal cell carcinoma. BJU Int 95: 421-424, 2005.

23. Takeda K, Smyth MJ, Cretney E, et al: Involvement of tumor necrosis factor-related apoptosis-inducing ligand in NK cellmediated and IFN-gamma-dependent suppression of subcutaneous tumor growth. Cell Immunol 214: 194-200, 2001. 